\title{
Un document inédit à propos de la mission du 9 mars 1793 pour la levée des 300000 hommes
}

\section{Michel Biard}

\section{(2) OpenEdition \\ 1 Journals}

\section{Édition électronique}

URL : https://journals.openedition.org/ahrf/1983

DOI : 10.4000/ahrf.1983

ISSN : 1952-403X

Éditeur :

Armand Colin, Société des études robespierristes

\section{Édition imprimée}

Date de publication : 1 septembre 2001

Pagination : 99-104

ISSN : 0003-4436

\section{Référence électronique}

Michel Biard, «Un document inédit à propos de la mission du 9 mars 1793 pour la levée des 300000 hommes », Annales historiques de la Révolution française [En ligne], 325 I juillet-septembre 2001, mis en ligne le 10 avril 2006, consulté le 24 avril 2022. URL : http://journals.openedition.org/ahrf/1983 ; DOI : https://doi.org/10.4000/ahrf.1983

Ce document a été généré automatiquement le 24 avril 2022.

Tous droits réservés 


\title{
Un document inédit à propos de la mission du 9 mars 1793 pour la levée des 300000 hommes
}

\author{
Michel Biard
}

1 Le 9 mars 1793, Carnot, au nom des comités de défense générale et de la guerre réunis, présentait devant la Convention nationale un rapport et un projet de décret tendant à envoyer dans les départements de la République des commissaires chargés d'« instruire leurs concitoyens des dangers qui menacent la patrie et rassembler des forces suffisantes pour disperser les ennemis ». Il s'agissait ainsi d'accélérer les mesures destinées à lever les 300000 « volontaires réclamés par l'Assemblée le 24 février ».

Cette mission collective fut l'acte fondateur de l'institution des représentants en mission. Là où la Convention s'était contentée jusqu'alors d'imiter les Assemblées précédentes en envoyant dans les départements ou aux armées des députés nantis d'une commission dans la plus pure tradition de l'Ancien Régime, elle créait au printemps 1793 une véritable institution qui devait durer jusqu'en brumaire an IV.

Les Archives parlementaires (tome LX, pp.9-10) donnent, bien entendu, le décret du 9 mars avec la liste des quarante et une sections qui devaient être parcourues par ceux qui furent dès avril appelés « représentants en mission » et non plus commissaires, ce qui marquait tout à la fois une volonté de les distinguer de la foule des autres commissaires, d'institutionnaliser la méthode et d'affirmer la légitimité des envoyés de la Convention. Ce même recueil contient la liste des quatre-vingt-deux représentants qui furent initialement choisis pour cette mission collective (tome LX, p.25). Ces conventionnels devaient être répartis par équipes de deux, chaque équipe étant chargée d'une section géographique associant deux départements voisins. Loin d'être le fruit d'un hasard lié à la plume d'un anonyme scribe de l'Assemblée, l'ordre dans lequel les noms furent couchés sur la liste était lourdement chargé de sens politique (cf. notre article, «La mission du 9 mars 1793 », dans Pour la Révolution française, recueil d'études réunies par Christine le Bozec et Éric Wauters en hommage à Claude Mazauric, Rouen, Publications de l'Université de Rouen, 1998, pp.273-280). Pour autant, au siècle dernier, 
Alphonse Aulard avouait sa perplexité devant la composition de cette liste et, comme d'autres historiens, s'en tenait à la thèse classique d'une manœuvre de la Gironde destinée à éloigner de Paris un grand nombre de Montagnards. Nous l'avons montré, cette thèse est une totale aberration, ce qui ne l'empêche nullement de poursuivre aujourd'hui sa déjà longue carrière dans l'historiographie (à titre d'exemple, cf. Patrice Gueniffey, La politique de la Terreur. Essai sur la violence révolutionnaire,1789-1794, Paris, Fayard, 2000, p.248). Au-delà de cette vulgate sans cesse servilement recopiée, la mission du 9 mars présentait un second «mystère » nulle liste ne semblait avoir été conservée indiquant de quelle manière la fusion s'était réalisée entre les noms des quatre-vingt-deux représentants et les quarante et une sections géographiques. Alphonse Aulard, dans son Recueil des actes du Comité de salut public (...) (tome II, pp. 298-317), reconnaissait n'avoir trouvé que trois décrets relatifs à la destination des représentants en mission et écrivait « Nulle part il n'y a une liste officielle des couples de commissaires et des sections qui leur furent attribuées. Pour savoir au juste quels conventionnels firent réellement partie de la mission du 9 mars 1793 et dans quels départements ils se rendirent, il faut retrouver d'abord leur correspondance (et il en est dont nous n'avons rencontré aucune lettre) et parcourir les pièces relatives aux missions dans les départements (Arch. nat., AF II 83 à 146). Nous sommes parvenus ainsi à reconstituer, sauf erreur, le tableau de cette mission si complexe ». L'erreur est en l'occurrence bien humaine et le tableau dressé par Aulard a justement induit en erreur plus d'un historien. Ainsi, pour la section 26, réservée à la Vendée et aux Deux-Sèvres, Aulard donne pour missionnaires Du Bois du Bais et Gasparin. Une note indique qu'ils ont été "plus tard» (formule d'une rare précision!) remplacés par Auguis et Carra, auxquels fut adjoint Bourbotte. Or non seulement Du Bois du Bais et Gasparin ne participent en rien à cette mission pour laquelle ils n'apparaissent même pas sur les listes initiales (ils sont en fait envoyés, tous deux, à l'armée du Nord le 4 avril 1793), mais, qui plus est, Auguis et Carra, eux, figurent bel et bien sur celles-ci. Une erreur similaire est commise par Aulard pour la section 30 (Maine-et-Loire, Sarthe) où il inscrit les noms de Goupilleau de Montaigu et de Richard, en indiquant en note que le premier a été remplacé " plus tard » par Choudieu. Or le nom de ce dernier est couché sur la liste du 9 mars, ainsi que celui d'un Goupilleau, mais il s'agit du cousin de Montaigu, Goupilleau de Fontenay. Goupilleau de Montaigu est alors en mission dans la principauté de Salm, avec Couthon et Michel, en vertu des décrets des 2 et 3 mars précédents. C'est lui qui est adjoint dans un second temps à la mission du 9 mars, et non Choudieu.

Ce ne sont certes là que des erreurs de détail, mais elles ont tout récemment encore entachées des ouvrages par ailleurs tout à fait sérieux. Il convient donc de les rectifier. Plus encore, il convient désormais de classer au chapitre des énigmes élucidées la question de l'existence ou de l'absence d'une liste indiquant dès mars 1793 les destinations géographiques des missionnaires. Cette liste existe aux Archives nationales, conservée dans les papiers du comité des inspecteurs de la salle (fond très riche et de toute évidence peu exploité), sous la cote $\mathrm{D}^{*} \mathrm{XXXV}^{\mathrm{C}} 6$ (à la date du 16 mars 1793). Si elle contient elle aussi quelques menues erreurs (dues pour l'essentiel à des coquilles de "ronds de cuir » peu attentifs), cette liste est à notre connaissance le seul document original et inédit qui permette l'analyse de la mission du 9 mars 1793. C'est bien pourquoi il nous a semblé utile d'en faire bénéficier les lecteurs des AHRF, d'autant qu'elle contient de précieuses indications sur les sommes qui étaient délivrées aux représentants du peuple qui partaient en mission. Les papiers du comité des 
inspecteurs de la salle permettent d'ailleurs de retrouver la trace de la plupart des missions jusqu'à la séparation de la Convention en brumaire an IV et de connaître le montant des fonds qui furent délivrés aux missionnaires à leur départ. Certes ces sommes ne représentent pas forcément la totalité de ce qui a été utilisé par un représentant en mission puisque celui-ci gardait toute latitude à tirer des fonds des départements (comme le prouve un nombre important de comptes rendus rédigés en l'an III qui font apparaître sommes allouées et sommes dépensées par les conventionnels en mission). Néanmoins l'évolution de ces sommes permet d'évaluer le coût moyen des missions, de mettre à jour les effets de la dépréciation de l'assignat (en 1793 ce sont environ 30 à 300000 livres qui sont ainsi versées chaque mois, soit une moyenne qui oscille entre 3000 et 5000 livres par missionnaire en vendémiaire an IV le total est porté à 1704205 livres, soit une moyenne de 48691 livres par tête), et de distinguer les représentants les plus "gourmands »: le 29 juin 1793 Rovère demande avant son départ la somme alors exceptionnelle de 20000 livres; le comité lui observe qu'il s'agit là d'une somme " très considérable ", mais " le citoyen Rovère ayant observé que le Comité de salut public l'avait chargé de mission à Lyon qui exigeait des dépenses extraordinaires, le comité n'a pas cru pouvoir refuser au citoyen Rovère sa demande [...] ». Le même jour, Cavaignac et Goupilleau de Fontenay partent, respectivement à l'armée des côtes de Brest et à celle des côtes de La Rochelle, avec l'un 3000 livres, l'autre 6000 livres... et, à part le cas particulier de Couthon et Maignet en août 1793 (avec 36.000 livres), il faut attendre la fin de thermidor an II pour voir le « record » de Rovère dépassé (avec 25 000livres adjugées à Niou), avant bien sûr que toute valeur finisse par devenir surréaliste (les 500000 livres obtenues par Fréron en vendémiaire an IV). Ces derniers chiffres rendent presque dérisoires les sommes versées pour la mission du 9 mars 1793 telles qu'elles apparaissent, à la date du 16 mars, dans le tableau ci-dessous.

5 NB L'orthographe de l'original a été respectée. Pour des raisons de clarté, nous avons par contre ajouté un quadrillage qui n'existait pas dans le document.

6 "Le comité autorise le citoyen Saurine [?] à délivrer les mandats suivants aux commissaires envoyés dans les départements ». 


\begin{tabular}{|c|c|c|c|}
\hline Noms des départements & Noms des commissaires & $\begin{array}{l}\text { Sommes } \\
\text { données }\end{array}$ & $\begin{array}{c}\text { Dont en } \\
\text { argent }\end{array}$ \\
\hline Nord et Pas-de-Calais & Carnot et Lesage Senault & 3000 & 600 \\
\hline Aisnes et les Ardennes & $S^{t}$ Just et de Ville & 6000 & \\
\hline Meuse et Marne & Pons et Thuriot & 6000 & 1000 \\
\hline Meurthe et Mozelle & Anthoine et Levasseur 2 & 6000 & 2000 \\
\hline Haut et Bas Rhin & Louis et Pfiger ${ }^{3}$ & 4000 & 1200 \\
\hline Doubs et Haute Saône & Michaud et Siblot & 3000 & \\
\hline Côte d'Or et le Jura & Prost et Leonard Bourdon & 8000 & 1000 \\
\hline Haute Marne et Vosges & Perrin ${ }^{4}$ et Roux 5 & 4000 & \\
\hline Ain, Saone et Loire ${ }^{6}$ & Pressavin et Reverchon & 3000 & \\
\hline Rhône et Loire, l'Isère & Amar et Merlinot? & 4000 & \\
\hline Hautes et Basses Alpes & Barras et Freron & 8000 & \\
\hline Var et Alpes Maritimes & Roubaud et Despinassy & 9000 & \\
\hline Le Gard et l'Herault & Bonnier et Vouland8 & 6000 & \\
\hline Bouches du Rhône et Drôme & Boisset et Bayle & 6000 & \\
\hline Ardeche et Lozére & Serviére et Gleizal & 6000 & \\
\hline Haute Loire et Cantal & Renaud $^{9}$ et la Coste 10 & 6000 & \\
\hline La Correze et He Vienne & Borie et Bordas & 6000 & \\
\hline Le Tarn et l'Aveiron & Bo et Chabot & 6000 & \\
\hline LAude et $\mathrm{H}^{\mathrm{e}}$ Garonne & Mailhe et Lachaux "I & 8000 & \\
\hline Arriége et Pirennées Orientales & Gaston et Fayau & 12000 & \\
\hline Hautes et Basse Pirennées & Ysabeau et Neveu & 8000 & 2000 \\
\hline Gers et les Landes & Dartigoête1/2 et Ichon & 8000 & \\
\hline Gironde, Lot et Garonne & Garreau13 et Paganel & 8000 & \\
\hline Lot et la Dordogne & $\mathrm{S}^{\mathrm{t}}$ André $14 \mathrm{et} \mathrm{la} \operatorname{coste}^{15}$ & 6000 & \\
\hline Charente, Charente Infe & Bernard 16 et Gimberteau & 8000 & \\
\hline Deux Sevres et la Vendée & Carra et Auguis & 5000 & \\
\hline Loire Infe et Mayenne & Foucher17 et Lavallée 18 & 8000 & \\
\hline Morbihan et Finisterre & Mailhaud 19 et Guermeur & 6000 & \\
\hline Cotes du Nord, Ille et Vilaine & Sevestre et Billaud Vareine ${ }^{20}$ & 6000 & \\
\hline la Sarthe, Maine et Loire & Rigard21 et Choudieu & 4000 & \\
\hline La Manche et l'Orne & Carpentier 22 et Bourdon 23 & 6000 & \\
\hline LEure et le Calvados & Duroi $^{24}$ et Jouenne 25 & 5000 & \\
\hline Seine Infe et la Somme & Pocholle et Saladin & 6000 & \\
\hline Oise, Seine et Marne & Lakanal26 et Mauduit27 & 4000 & \\
\hline Eure et Loire, Seine et Oise & Chales et Levasseur ${ }^{28}$ & 3000 & \\
\hline Loire et Cher, Indre et Loire & Tallien et Goupilleau ${ }^{29}$ & 5000 & \\
\hline Indre et Vienne & Piory ${ }^{30}$ et Lejeune ${ }^{31}$ & 6000 & \\
\hline Creuse et Puy de Dôme & Monnestier 32 et Petitjean & 4000 & \\
\hline Cher et l'Allier & Forestier et Faure 33 & 3000 & \\
\hline & La Planche ${ }^{34}$ et Collot $^{35}$ & 5000 & \\
\hline \multirow{2}{*}{ Aube et I'Yonne } & Thureau $^{36}$ et Garnier ${ }^{37}$ & 6000 & \\
\hline & Total & 240000 & 7800 \\
\hline
\end{tabular}

1. Deville, représentant de la Marne

Le Vasseur de la Meurthe

3.1l convient bien sûr de lire Pflieger, représentant du Haut-Rhir

4. Perrin, représentant des Vosges.

6. Roux, représentant de la Haute-Marne.
y la une confusion avec la ligne suivante. Cette section

associe la Saône-et-Loire au Rhône-et-Loire, la suivante regroupe l'Ain et P' Isère.

associe la Saône-et-Loire au Rher
${ }^{7}$ Merlino, repré sentant de l'Ain

8. Voulland, représentant du Gard

'II s'agit de Reynaud, représentant de la Haute-Loire.

Celui-ci est d' ailleurs remplacé le 12 mars par Balthazar Faure

(lui aussi représentant de la Haute-Loire), ce qui n'est donc pas connu

du scribe qui rédige cette liste. Cela tend à prouver que si le comité

des inspecteurs de la salle traite cette affaire le 16 mars,

Iraisonne néanmoins sur la base d'une liste dressée antérieurement

${ }^{10}$ Jean-Baptiste Lacoste, représentant du Cantal

11. Lombard-Lachaux, représentant du Loiret.

${ }^{12}$ Dartigoeyte, représentant des Landes.

13.Garrau, représentant de la Gironde.

14.Jeanbon Saint-André, représentant du Lot

Elie Lacoste, representant de la Dordogie.

${ }^{16}$ Bemard de Saintes, représentant de la Charente-Inférieure.

17. Il faut évidemment lire Fouché, représentant de la Loire-Inférieure

${ }^{18}$ Esnuë-Lavallée, représentant de la Mayenne, est en fait

immédiatement remplacé par Villers, représentant de la Loire-Inférieure.

- Lire Le Malliaud, représentant du Morbihan

${ }^{20}$ Billaud-Varenne, représentant de Paris

Lire Richard, représentant de la Sarti

${ }^{22}$ Le Carpentier de la Manche

24 Dourdon de l Oise.

U

The reprentant du Calvados, est en fait

immédiatement remplacé par Bonnet de Meautry, lui aussi représentant du Calvados.

${ }^{26}$ Remplacé par Isoré le 25 mars

27. Mauduyt, représentant de Seine-et-Marne

29. Goupilleau de Fontenay, représentant de Vendée.

30. Piorry, représentant de la Vienne

${ }^{32}$ Monestier due puy-de de l'Indre.

33.II s'agit non de Faure, mais de Fauvre-Labrunerie, repré sentant du Cher

34. Goyre-Laplanche, représentant de la Nievre.

${ }^{35}$ Collot d'Herbois, représentant de Paris.

${ }^{36}$ Turreau, représentant de l'Yonne.

37. Gamier de l'Aube. 


\section{AUTEUR}

MICHEL BIARD

Institut d'Histoire de la Révolution française 